\title{
Cosmic re-onization: theoretical modelling and forthcoming observations
}

\author{
Benedetta Ciardi \\ Max-Planck-Institut fr̈ Astrophysik, Karl-Schwarzschild-Straße 1, D-85741 Garching, Germany \\ email: bciardi@mpa-garching.mpg.de
}

\begin{abstract}
With the advent in the near future of radio telescopes such as LOFAR and the SKA, a new window on the high-redshift Universe will be opened. In particular, it will be possible, for the first time, to observe the $21-\mathrm{cm}$ signal from the diffuse IGM prior to its complete reionization and thus probe the 'dark ages'. I discuss the theoretical modelling of the re-ionization process and its observability through the 21-cm signal and the CMB anisotropies.
\end{abstract}

Keywords. atomic processes, line formation, cosmology: observations, cosmology: theory

In the past few years, the study of the intergalactic medium (IGM) re-ionization has attracted considerable attention and has moved from being a purely theoretical excercise to an observational goal. At present, the only available observations on the re-ionization process are those of high-redshift quasar spectra (which provide information on the abundance of neutral hydrogen during the last stage of the process at $z \operatorname{sim} 6$; e.g., Fan et al. 2004) and of anisotropies in the power spectrum of the Cosmic Microwave Background (CMB) radiation (which give an estimate of the global amount of electrons produced by re-ionization; Spergel et al. 2007). Despite the most recent progress though, we still lack observations that probe the temporal evolution of the re-ionization process and that answer some still very debated questions: What are the first sources of ionizing radiation? How did re-ionization evolve? Which is its interplay with the galaxy formation process? The perfect tool to start answering these questions is the 21-cm line associated with the hyperfine transition of the ground state of $\mathrm{H}$ I. Observations of the 21-cm line at different frequencies can give information on the state of $\mathrm{H}$ I at different redshifts. With the advent in the near future of radio telescopes such as LOFAR, MWA, and the SKA, a new window on the high-redshift Universe will be opened and it will be possible, for the first time, to probe the re-ionization history and the 'dark ages'.

From a theoretical point of view, the ingredients needed to model the re-ionization process are: $(i)$ simulations of galaxy formation; $(i i)$ properties of the ionization sources; and (iii) radiative transfer (RT) of ionizing photons in the IGM. The latter point is particularly tricky given the complexity of the equations involved and the present impossibility of solving them. For this reason, several groups are working on the development of RT codes based on different assumptions and approximations (for a comparison between the RT codes present in the cosmological community, see Iliev et al. 2006).

We have run numerical simulations of the re-ionization process in a box of comoving length $20(10) h^{-1} \mathrm{Mpc}$ representing a 'mean' ('proto-cluster') region of the Universe. The sources are stars with variable properties (Ciardi et al. 2003a; Ciardi et al. 2003b) and the radiative transfer has been followed using the code CRASH (Ciardi et al. 2001; Maselli et al. 2003). The simulations have been run also with the addition of sub-grid physics to take into account the effect of mini-halos (MHs, Ciardi et al. 2006). Figure 1 shows an example. The main results can be summarized as follows: $(i)$ the environment can have a deep impact on re-ionization; ( $i i)$ the presence of MHs can delay re-ionization up 


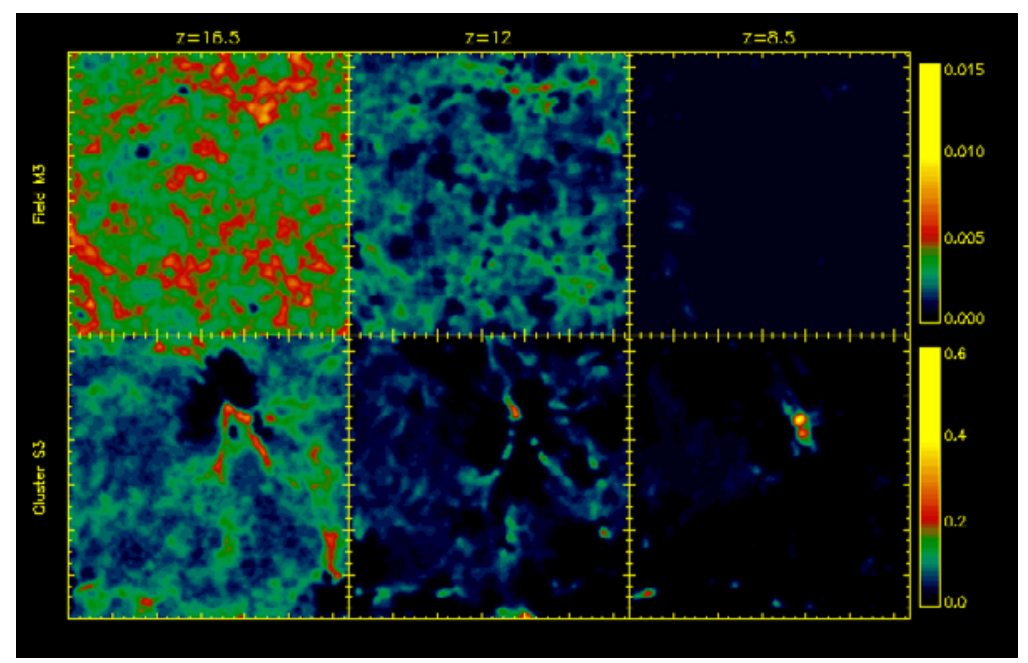

Figure 1. Slices cut through the simulation boxes. The panels show the H I number density for the 'mean' region of the Universe (upper) and the proto-cluster (lower) at $z=16.5,12$, and 8.5.

to $\Delta z \simeq 2$; and (iii) a suitable choice of the parameters involved in the calculations can reproduce the Thomson scattering optical depth measured by WMAP1 and WMAP3 (Spergel et al. 2007). The latter point suggests that to better contrain the theoretical modelling, additional observations are needed.

Ideal observations will be those of the 21-cm line from H I. If the IGM has been heated, the line is observed in emission and its intensity (described by the brightness temperature, $T_{\mathrm{b}}$ ) is directly proportional to the abundance of $\mathrm{H}$. Thus, it is straightforward to produce maps of 21-cm line emission from those of $\mathrm{H}$ I obtained from the above simulations. From the analysis of such maps we can conclude that (Ciardi \& Madau 2003; Salvaterra et al. 2005; Valdes et al. 2006): (i) fluctuations of $T_{\mathrm{b}}$ show a behaviour almost independent of the re-ionization history, with a peak at $\sim 10 \mathrm{mK}$ (earlier re-ionization peaks at frequencies lower than a later re-ionization); (ii) the expected emission is within the sensitivity range of the next generation of radio telescopes; (iii) 21-cm observations can be used in combination with other measurements (i.e., CMB) to maximize the extracted information; and $(i v)$ the greatest challenge will be dealing with foreground contamination of both Galactic and extragalactic origin.

\section{Acknowledgements}

I thank my collaborators in these projects.

\section{References}

Ciardi, B., Ferrara, A., Marri, S., \& Raimondo, G. 2001, MNRAS, 324, 381

Ciardi, B., Ferrara, A., \& White, S. D. M. 2003, MNRAS (Letters), 344, L7

Ciardi, B., \& Madau, P. 2003, ApJ, 596, 1

Ciardi, B., Scannapieco, E., Stoehr, F., et al. 2006, MNRAS, 366, 689

Ciardi, B., Stoehr, F., \& White, S. D. M. 2003a, MNRAS, 343, 1101

Fan, X., Hennawi, J. F., Richards, G. T., et al. 2004, AJ, 128, 515

Iliev, I., Ciardi, B., Alvarez, M. A., et al. 2006, MNRAS, 371, 1057

Maselli, A., Ferrara, A., \& Ciardi, B. 2003, MNRAS,345, 379

Salvaterra, R., Ciardi, B., Ferrara, A., \& Baccigalupi, C. 2005, MNRAS, 360, 1063

Spergel D.N., Bean, R., Dor, O.,et al. 2007, ApJS, 170, 377

Valdes, M., Ciardi, B., Ferrara, A., Johnston-Hollitt, M., \& Röttgering, H. 2006, MNRAS (Letters), 369, L66 